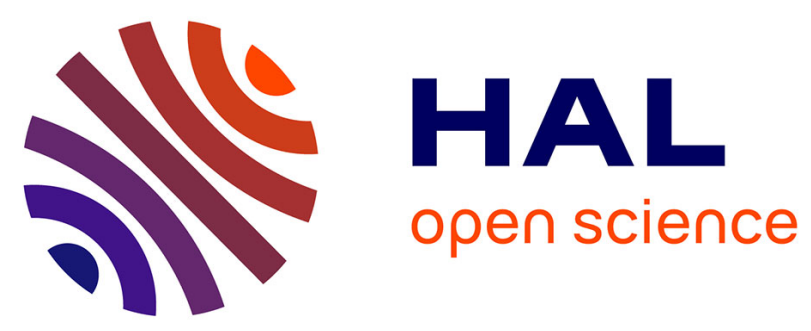

\title{
Temperature-Aware Density Optimization for Low Power Wireless Sensor Networks
}

Abdelmalik Bachir, Walid Bechkit, Yacine Challal, Abdelmadjid Bouabdallah

\section{To cite this version:}

Abdelmalik Bachir, Walid Bechkit, Yacine Challal, Abdelmadjid Bouabdallah. Temperature-Aware Density Optimization for Low Power Wireless Sensor Networks. IEEE Communications Letters, 2013, 17 (2), pp.325-328. 10.1109/LCOMM.2013.011113.122286 . hal-00799905

\section{HAL Id: hal-00799905 https://hal.science/hal-00799905}

Submitted on 12 Mar 2013

HAL is a multi-disciplinary open access archive for the deposit and dissemination of scientific research documents, whether they are published or not. The documents may come from teaching and research institutions in France or abroad, or from public or private research centers.
L'archive ouverte pluridisciplinaire HAL, est destinée au dépôt et à la diffusion de documents scientifiques de niveau recherche, publiés ou non, émanant des établissements d'enseignement et de recherche français ou étrangers, des laboratoires publics ou privés. 


\title{
Temperature-Aware Density Optimization for Low Power Wireless Sensor Networks
}

\author{
Abdelmalik Bachir, Walid Bechkit, Yacine Challal, Abdelmadjid Bouabdallah
}

\begin{abstract}
High temperatures negatively affect the quality of radio communication links both at transmission and reception sides. In this paper, we investigate the effect of temperature on coverage and connectivity and show that more energy can be saved by allowing some nodes to go to deep sleep mode when temperature decreases and links improve. We propose a simple and fully distributed temperature-aware algorithm that dynamically adapts the network effective density to allow further energy savings while maintaining network connectivity and full coverage.
\end{abstract}

Index Terms-Wireless Sensor Networks, Temperature Impact, Connectivity, Energy Saving.

\section{INTRODUCTION}

Wireless sensor networks use low power transceivers to enable nodes to communicate at low energy cost. These transceivers are sensitive to temperature variations which affect both their transmission and reception capabilities. The effect of temperature has been validated independently by many researchers in the literature (e.g. [1] [2]), and documented in the data sheets of the low power radios (e.g. [3]). As shown in Fig. 1, when temperature increases, both transmission power and sensitivity decrease which leads to the degradation of wireless communication links, and thus the overall connectivity of the network. As an example, a network designed to operate under typical conditions, i.e. a temperature of $25^{\circ} \mathrm{C}$, will experience connectivity degradation when temperature increases above $25^{\circ} \mathrm{C}$. Conversely, when temperature decreases below $25^{\circ} \mathrm{C}$, wireless link quality improves, the network becomes over-dimensioned and some nodes can go to deep sleep mode without affecting the connectivity of the network.

In this paper, we exploit the fact that low temperatures result in over-dimensioned networks, and propose to save further energy by allowing those nodes which are not necessary for the correct operation of the network to go to deep sleep mode when temperature is low and resume their normal functioning when temperature is high. We assume that a network continues to operate correctly if both full coverage and connectivity are guaranteed. A full coverage means that the entire deployment area is fully covered so that no event (e.g. environmental phenomenon) will be missed, and a connected network means that there is a route between any nodes in the network so that sensed data can be reported to any node of the network.

Abdelmalik Bachir is with EEE Department, Imperial College London, UK. E-mail: a.bachir@imperial.ac.uk. Walid Bechkit, Yacine Challal, Abdelmadjid Bouabdallah are with Université de Technologie de Compiègne, HEUDIASYC UMR CNRS 7253, Compiègne, France. E-mails: \{wbechkit, ychallal, bouabdal\}@hds.utc.fr. This work is partially supported by Picardie/ERDF AgroSens project.

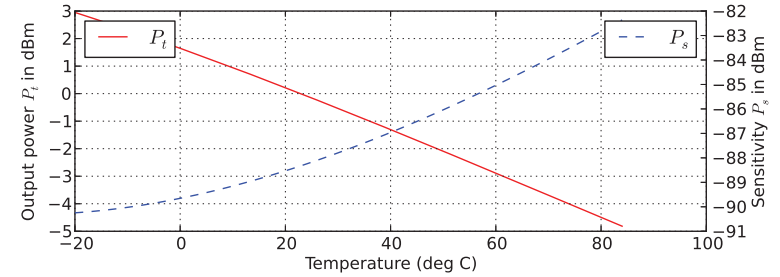

Fig. 1. Effect of temperature variations on the transmit power (targeted 0 $\mathrm{dBm}$ ) and sensitivity of the receiver for the CC2400. The figures have been generated by extrapolation from values of typical temperatures provided in the data sheet [3].

\section{System Model}

\section{A. Wireless Links}

The quality of a communication link is generally expressed by the experienced PER (Packet Error Rate). The PER mainly depends on the signal strength measured at the receiver. The minimum signal strength required to achieve a PER of less than $1 \%$ is called sensitivity. We assume that a link exists if the received power $P_{r}$ is equal or larger than the sensitivity $P_{s}$.

The signal strength measured at the receiver depends on the transmission power $P_{t}$ (also known as the output power) radiated by the transmitter and its attenuation along the communication channel. The attenuation $a(d)$ over a distance $d$ is the ratio between $P_{t}$ and $P_{r}(d)$ where $P_{r}(d)$ is the power of the signal received at distance $d$ from the transmitter. When $P_{t}$ and $P_{r}(d)$ are expressed in $\mathrm{dBm}$, the attenuation in $\mathrm{dB}$ is:

$$
a(d)=P_{t}-P_{r}(d)
$$

The attenuation has two components: $a_{1}(d)$ and $a_{2}$ such that:

$$
a(d)=a_{1}(d)+a_{2}
$$

where $a_{1}(d)$ and $a_{2}$ are defined as:

$$
\begin{cases}a_{1}(d) & =\alpha 10 \log \left(d / d_{\text {ref }}\right) \\ a_{2} & \sim \mathcal{N}(0, \sigma)\end{cases}
$$

where $\alpha$ is the path loss exponent (usually taken between 2 and 4) and $d_{\text {ref }}$ is the reference distance which is typically taken as 1 meter. The component $a_{2}$ does not depend on distance and follows a normal distribution $\mathcal{N}(0, \sigma)$, where $\sigma$ is in $\mathrm{dB}$.

We define the communication range as the distance at which less than $1 \%$ of packets are lost (i.e. corresponding to less than $1 \%$ of PER). The communication range is a random variable $R$. We use the notation $R(d)$ to express the probability that the transmission range is equal or larger than $d$, i.e. $R(d)=$ $\operatorname{Pr}[R \geq d]$, which is equivalent to $\operatorname{Pr}\left[P_{r}(d) \geq P_{s}\right]$, which 
can also be written as $\operatorname{Pr}\left[P_{t}-P_{r}(d) \leq P_{t}-P_{s}\right]$. In terms of attenuation, we have:

$$
R(d)=\operatorname{Pr}\left[a(d) \leq a_{s}\right]
$$

where $a_{s}$ is the maximum attenuation that allows $99 \%$ of packet reception, i.e. $a_{s}=P_{t}-P_{s}$. By replacing $a(d)$ by its value, (4) can be rewritten as:

$$
\begin{aligned}
R(d) & =\operatorname{Pr}\left[a_{1}(d)+a_{2} \leq a_{s}\right] \\
& =\operatorname{Pr}\left[a_{2} \leq a_{s}-a_{1}(d)\right]
\end{aligned}
$$

As $a_{2}$ is a normal distribution, $R(d)$ is given by:

$R(d)= \begin{cases}\frac{1}{2}+\frac{1}{2} \operatorname{erf}\left(\frac{P_{t}-P_{s}-10 \alpha \log d}{\sqrt{2} \sigma}\right) & \text { if } \sigma>0, \\ 1_{(-\infty, \mathcal{R}]}(d) & \text { if } \sigma=0 .\end{cases}$

where $\mathcal{R}$ is the communication range in the abscence of shadowing fading (i.e. when $\sigma=0$ ), given by the following:

$$
\mathcal{R}=10^{\left(P_{t}-P_{s}\right) / 10 \alpha}
$$

and erf is the error function defined as:

$$
\operatorname{erf}(z)=\frac{2}{\sqrt{\pi}} \int_{0}^{z} e^{-t^{2}} d t
$$

and $1_{(-\infty, \mathcal{R}]}(d)$ is the indicator function, which is equal to 1 when $d \leq \mathcal{R}$ and to 0 elsewhere.

Note that the previous derivations also apply to the two ray ground model which may be more appropriate for outdoor environments. The results for the two ray ground model can be obtained by taking $\sigma=0$ in (7) and $\mathcal{R}=$ $\left(G_{t} G_{r} H_{t}^{2} H_{r}^{2}\right)^{1 / 4} 10^{\left(P_{t}-P_{s}\right) /(4 \times 10)}$ in (8), where $G_{t}$ (resp. $\left.G_{r}\right)$ is the antenna gain at the transmitter (resp. receiver), and $H_{t}$ (resp. $H_{r}$ ) is the antenna height at the transmitter (resp. receiver).

\section{B. Network Connectivity and Coverage}

We consider the case where nodes are deployed according to a PPP (Poisson Point Process). Under these circumstances, the probability $p_{\text {cov }}$ that the deployment area is fully covered can be approximated to the following [8]:

$$
p_{\text {cov }}=1-p_{i}
$$

where $p_{i}$ is the node isolation probability defined as [5]:

$$
p_{i}=e^{-\lambda \pi R_{s}^{2}}
$$

where $\lambda$ is the density of nodes per unit area, and $R_{s}$ is the sensing range, i.e. all events occurring at a distance smaller than $R_{s}$ can be sensed by the node.

The probability $p_{c}$ that the network is connected has been calculated in [7], and is equal to:

$$
p_{c}=e^{-\lambda \mu P_{I}}
$$

where $\mu$ is the surface of the deployment area, and $P_{I}$ is defined as:

$$
P_{I}=e^{-\lambda \pi R_{s}^{2} e^{(\sqrt{2} \sigma / \alpha)^{2}}}
$$

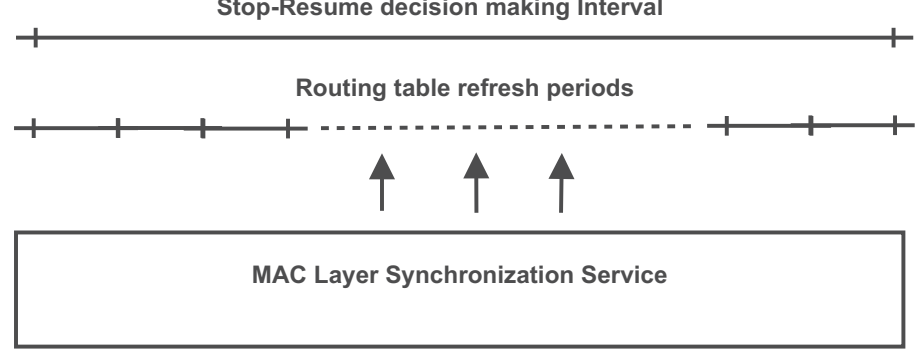

Fig. 2. Synchronization assumptions and requirements

\section{Synchronization Assumptions}

As it will be explained later, nodes will run, periodically, a stop-resume decision making algorithm. This requires synchronization of nodes' clocks. We assume that our algorithm relies on the synchronization provided by the underlying MAC protocol such as: SCP-MAC [9] and IEEE 802.15.4 [6] which could be rather loose since our algorithm tolerates a clock drift up to one stop-resume decision making interval. In this case, the configuration will not have side effect on the algorithm stabilization but would be sub-optimal during eventual negligible clock drift.

Furthermore, in order to avoid any side effect on routing protocols, the stop- resume decision making interval must be longer than the routing table refresh period. Since temperature change is relatively slow, this assumption will be reasonable. In addition, routing table refresh interval must be aligned with the starting point of the stop-resume decision making period. Consequently, routing protocols will take into consideration the new configuration seamlessly without extra-overhead.

In Fig. 2, we summarize the synchronization assumptions and requirements for a smooth operation of our scheme.

\section{EFFECT OF TEMPERATURE}

\section{A. On Wireless Links}

We use the notation $P_{t}\left(\tau_{t}\right)$ (resp. $P_{s}\left(\tau_{r}\right)$ ) to express the variation of the transmission power (resp. sensitivity) in function of the temperature $\tau_{t}$ (resp. $\tau_{r}$ ) measured at the transmitter (resp. receiver). Although no closed-form expression for $P_{t}\left(\tau_{t}\right)$ (resp. $\left.P_{s}\left(\tau_{r}\right)\right)$ is provided, the data provided in the data sheet allows to find an approximate polynomial representations for $P_{t}\left(\tau_{t}\right)$ and $P_{s}\left(\tau_{r}\right)$ (see Fig. 1). We use $\hat{P}_{t}$ (resp. $\hat{P}_{s}$ ) to refer to the approximation of $P_{t}$ (resp. $P_{s}$ ). Fig. 3, plotted with values from $\hat{P}_{t}$ and $\hat{P}_{s}$, shows the drastic reduction in link quality for three sets of temperatures $20^{\circ} \mathrm{C}, 40^{\circ} \mathrm{C}$, and $60^{\circ} \mathrm{C}$, considering that the temperatures measured at the transmitter and the receiver are the same.

\section{B. On Coverage and Network Connectivity}

As shown in equations (10) to (13), both coverage and connectivity depend on the initial deployment $\lambda$. The connectivity further depends on temperature indirectly as the latter affects the transmission range $\mathcal{R}$. In Fig. 4, we fix the 


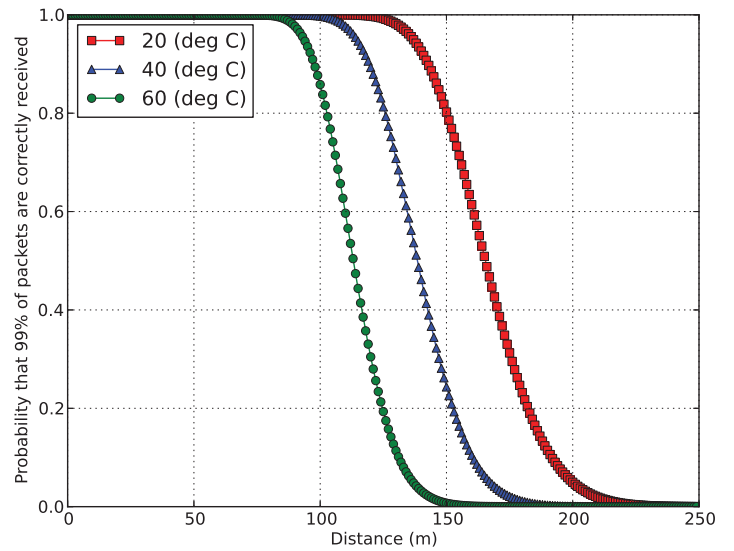

Fig. 3. The effect of temperature on wireless links in the presence of shadowing fading $(\sigma=2)$ and with a path loss exponent $\alpha=4$. The radio used is the $\mathrm{CC} 2400$ with a targeted output power of $0 \mathrm{dBm}$. The figure shows that higher temperatures reduce the probability that packets are correctly received at farther distances from the transmitter, which can be grossly interpreted as lower transmission ranges.

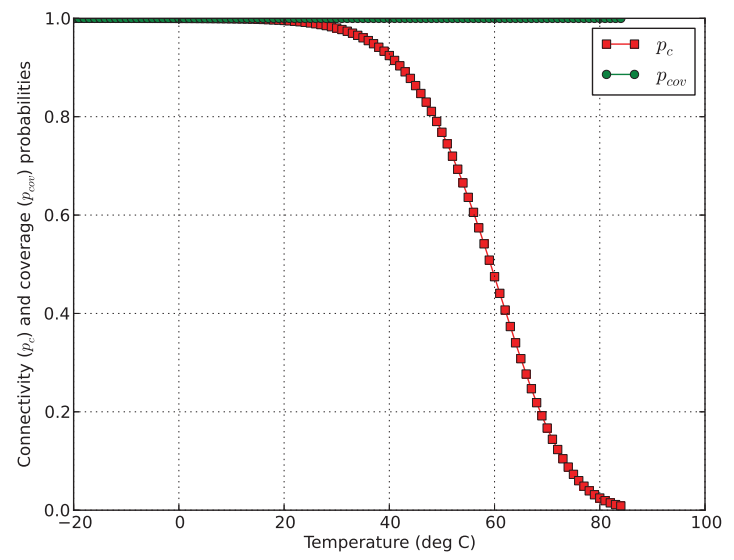

Fig. 4. The effect of temperature on coverage and network connectivity. The temperature interval ranges from $-20^{\circ} \mathrm{C}$ to $85^{\circ} \mathrm{C}$ which is the operating range of the CC2400 as described in [3]. The surface of the deployment area $\mu$ is $1000 m \times 1000 m$. Under these circumstances $\lambda_{\tau^{*}}$ is equal to $6.8 \times$ $10^{-5} m^{-2}$. The sensing range $R_{s}$ used for the calculation of $p_{\text {cov }}$ was taken to be equal to $\mathcal{R}$.

initial deployment to $\lambda_{\tau^{*}}{ }^{1}$ to the one that achieves a $99 \%$ connectivity in typical conditions (i.e. $25^{\circ} \mathrm{C}$ ), the sensing range to the typical transmission range (i.e. $\mathcal{R}$ at $25^{\circ} \mathrm{C}$ ), and vary the temperature while assuming it is homogeneous over the deployment area. Fig. 4 shows that while temperature increase significantly deteriorates connectivity, coverage remains guaranteed. Therefore, under this configuration it is sufficient to ensure connectivity to guarantee the correct operation of the network: connectivity and coverage.

${ }^{1}$ This value needs to be calculated numerically by combining (12) and (13)

\section{TEMPERATURE-AWARE DENSITY OPTIMIZATION}

We use $\tau^{+}$(resp. $\tau^{-}$) to refer to the maximum (resp. minimum) temperature. As temperature changes, the minimum density of nodes to ensure connectivity needs to change accordingly, i.e. $\lambda_{\tau^{-}}$at temperature $\tau^{-}$and $\lambda_{\tau^{+}}$at temperature $\tau^{-}$. To ensure connectivity in the worst case, the density of nodes should be equal to $\lambda_{\tau^{+}}$. However, when temperature decreases, a lower density is sufficient. We propose to calculate $\rho_{\tau}=\lambda_{\tau} / \lambda_{\tau^{+}}$which represents the ratio of network density that is required to maintain connectivity at temperature $\tau$ and an initial density of $\lambda_{\tau^{+}}$. The value of $\rho_{\tau}$ also expresses the number of nodes required to operate normally at temperature $\tau$ compared to those initially deployed (those which operate normally at temperature $\tau^{+}$), as $\rho_{\tau}=\frac{\lambda_{\tau}}{\lambda_{\tau}+}=\frac{\lambda_{\tau} \mu}{\lambda_{\tau}+\mu}$.

The basic idea of our approach is to keep the ratio of nodes which are operating normally equal to $\rho_{\tau}$ at temperature $\tau$. Those nodes which are not needed can go to deep sleep mode. The selection of those nodes which can stop operating can be done randomly, because the distribution of nodes which continue to operate normally maintains the same characteristics, i.e. distributed according to PPP. By assuming that each node is able to know its temperature (temperature sensor readings, extraction from log files, computation, expectation, etc.), we propose Algorithm 1 to allow unnecessary nodes stop operating when temperature decreases and resume operating when temperature increases. Algorithm 1 is fully distributed, and each node can run it periodically without exchanging any additional information with its neighbors. For efficiency and practicality, the values of $\rho_{\tau}$ need to be stored in table ${ }^{2}$ to avoid complex computation at the sensor node.

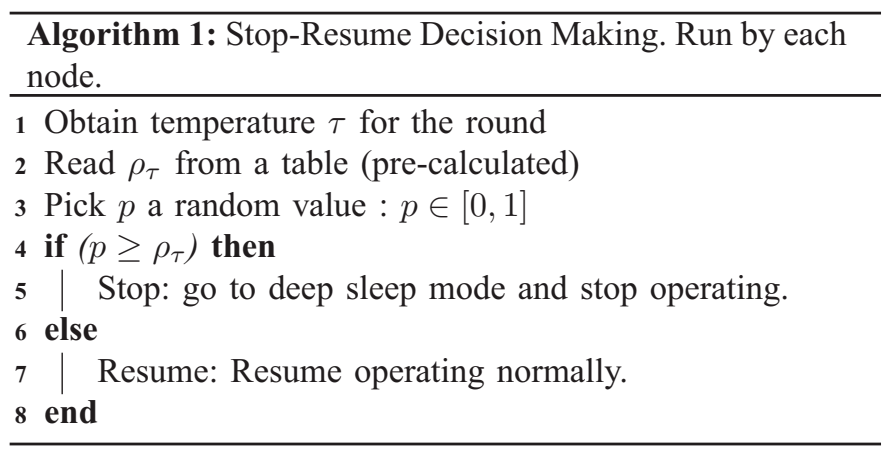

\section{Evaluation}

The mean power saved with the use of Stop-Resume algorithm at temperature $\tau$ compared to the traditional case where no temperature-aware mechanism is used is equal to $S(\tau)$ defined as:

$$
S(\tau)=1-\frac{\rho_{\tau} \mathcal{P}+\left(1-\rho_{\tau}\right) \mathcal{P}_{\mathrm{ds}}}{\mathcal{P}}=\left(1-\rho_{\tau}\right)\left(1-\frac{\mathcal{P}_{\mathrm{ds}}}{\mathcal{P}}\right)
$$

\footnotetext{
${ }^{2}$ The size of the table depends on the granularity of the temperature interval partitioning. In a case where temperature would vary from $0^{\circ} \mathrm{C}$ to $49^{\circ} \mathrm{C}$, and where each variation of $1^{\circ} \mathrm{C}$ has its corresponding $\rho$, the size of the table would be $50 \times 4=200$ bytes because $\rho_{\tau}$ is encoded as float which takes 4 bytes.
} 
where $\mathcal{P}$ is the mean power consumed when a node is running in the traditional case (which includes low power MAC protocols with duty-cycling) and $\mathcal{P}_{\mathrm{ds}}$ is the mean power consumed during deep sleep mode ${ }^{3}$. The term $\mathcal{P}_{\mathrm{ds}} / \mathcal{P}$ is generally very small compared to 1 and thus can be neglected. Therefore, the mean power saved by using Stop-Resume can be approximated to $\rho_{\tau}$, which does not depend on the used MAC protocol as long as $\mathcal{P}_{\mathrm{ds}} / \mathcal{P}$ remains very small compared to 1 .

Figure 5 shows the mean power saved by a node running Stop-Resume compared to the traditional case where no temperature aware deep sleep is implemented. We consider two cases for initial deployments: (i) at a typical $25^{\circ} \mathrm{C}$, and (ii) at the worst case temperature $38^{\circ} \mathrm{C}$ according to Erbs model [4]. For the typical case deployment, it shows how the connectivity of the traditional case improves when temperature is lower than the typical $25^{\circ} \mathrm{C}$ and how it deteriorates when temperature is higher than the typical $25^{\circ} \mathrm{C}$. With Stop-Resume, the connectivity does not go beyond the preset threshold of $99 \%$ of connectivity because those nodes which would increase connectivity would go to deep sleep mode. The same behavior (connectivity in function of temperature) is observed for the worst case deployment, but with a noticeable mean power saving with Stop-Resume, because the amount of nodes which would go to deep sleep mode is larger compared to the typical case where the amount of nodes deployed initially is smaller.

To evaluate the amount of energy that would be saved by using Stop-Resume, we evaluate the mean power consumed (i.e. the mean energy drained per second) by using StopResume compared to the traditional case with both typical and worst case deployments by integrating over a 24 hours interval, as shown below:

$$
\int_{24 \mathrm{~h}} S(\tau(t)) d t \approx \int_{24 \mathrm{~h}}\left(1-\rho_{\tau(t)}\right) d t
$$

By using the expression of temperature variation in function of time defined in Erbs model [4], the mean power saved by using Stop-Resume is around $7.86 \%$ in the typical deployment, and $22.32 \%$ in the worst case deployment.

\section{CONCLUSIONS AND FUTURE WORKS}

We showed that temperature variations impact the performance of wireless sensor networks significantly. We presented a simple yet effective method to take advantage of temperature variations to make the network save more energy while still achieving the preset performance requirement, in particular in terms of connectivity and coverage. The current work is limited to homogeneous networks, where both density and temperature are constant over the deployment area. While the thermal homogeneity is valid to a large extent particularly in outdoor applications, the density case should require further investigations. Although we believe that there are a number of applications in which the deployment would be densityhomogeneous by design, our proposal remains applicable in the general case provided that the deployment area can be

\footnotetext{
${ }^{3}$ Note that $\mathcal{P}_{\mathrm{ds}}$ is very small but not equal to zero, because the node keeps running essential components such as some of the timers to allow itself to resume operating.
}
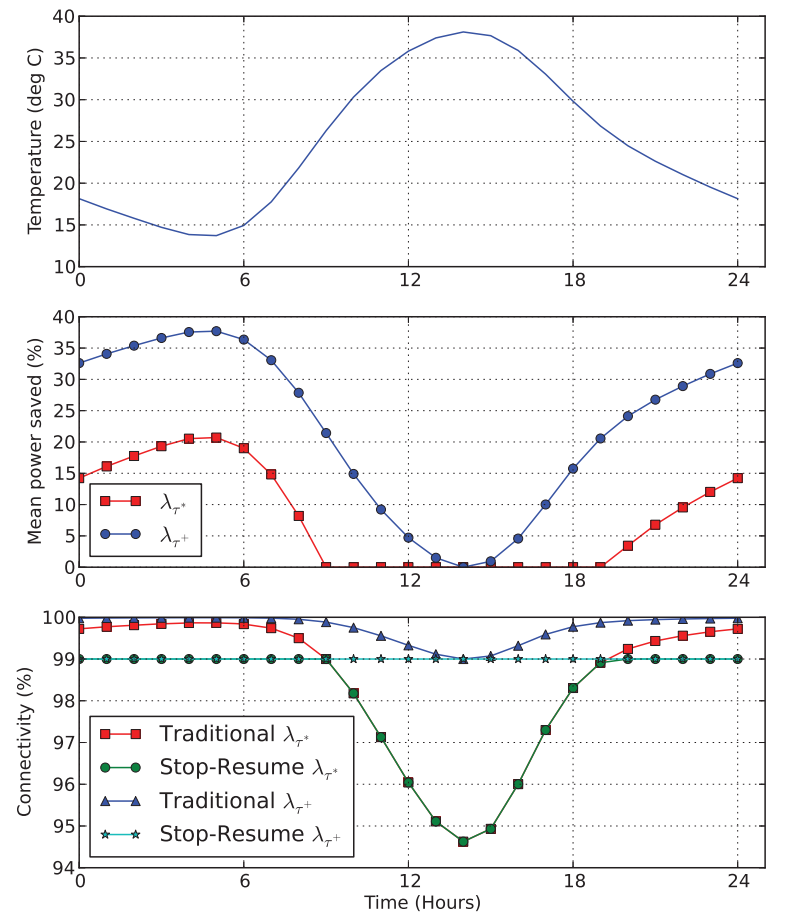

Fig. 5. Top: Temperature variations according to Erbs model [4]. Middle: The mean power saved by using Stop-Resume compared to the traditional case. Bottom: Connectivity of the network.

divided into homogenous sub-areas. With this division, each node can run Stop-Resume according to the density of the sub-area it belongs to. A future work will follow this direction and will investigate how the division into homogenous density sub-areas can be done efficiently and in a distributed way.

\section{REFERENCES}

[1] K. Bannister, G. Giorgetti, and S. Gupta, "Wireless sensor networking for hot applications: Effects of temperature on signal strength, data collection and localization," in Proceedings of the 5nd IEEE workshop on Embedded Networked Sensors, Charlotteville, VA, USA, 2008.

[2] C. A. Boano, J. Brown, N. Tsiftes, U. Roedig, and T. Voigt, "The impact of temperature on outdoor industrial wsn applications," IEEE Trans. Industrial Informatics, vol. 6, no. 3, pp. 451-459, 2010.

[3] C. Corporation, "CC2400 $2.4 \mathrm{GHz}$ Low-Power RF Transceiver, Data Sheet," 2008. [Online]. Available: http://www.ti.com/lit/gpn/cc2400

[4] D. G. Erbs, "Models and applications for weather statistics related to building heating and cooling loads." Ph.D. dissertation, Mechanical Engineering Dept., University of Wisconsin Madison,, 1984.

[5] P. Hall, Introduction to the Theory of Coverage Processes. NY, USA: J. Wiley and Sons, 1988.

[6] IEEE 802.15.4, "Wireless Medium Access Control (MAC) and Physical Layer (PHY) Specifications for Low-Rate Wireless Personal Area Networks (LR-WPANs)," 2003.

[7] D. Miorandi, "The impact of channel randomness on coverage and connectivity of ad hoc and sensor networks," Trans. Wireless. Comm., vol. 7, no. 3, pp. 1062-1072, Mar. 2008. [Online]. Available: http://dx.doi.org/10.1109/TWC.2007.060842

[8] D. Stoyan, W. S. Kendall, and J. Mecke, Stochastic Geometry and its Applications. NY, USA: J. Wiley and Sons, 1987.

[9] W. Ye, F. Silva, and J. Heidemann, "Ultra-low duty cycle mac with scheduled channel polling," in Proceedings of ACM SenSys, 2006, pp. 321-334. 